\title{
Acute orbital myositis before Herpes zoster ophthalmicus
}

\author{
Hyung Tae Kim, Soo Young Moon, and Ki Hyun Lee \\ Department of Anesthesiology and Pain Medicine, Presbyterian Medical Center, Jeonju, Korea
}

In the literature, $7 \%$ of all cases of herpes zoster present as herpes zoster ophthalmicus (HZO). Of these cases, 20 to $79 \%$ have orbital involvement [1]. Nearly all orbital tissues, including extra-ocular muscles, can be affected by the varicella zoster virus. Orbital involvement may present as keratitis, uveitis, scleritis, optic neuritis, ocular motor palsy, or postherpetic neuralgia (PHN) [2]. HZO is often suspected when ophthalmic symptoms and signs follow characteristic skin rashes and edema. Here, we report an unusual case where orbital myositis preceded vesicular skin eruptions.

A 66 year-old man, who had diabetes for 15 years and hypertension for 10 years, was hospitalized due to a 4-day history of left orbital shooting pain. Cranial nerve examination was nearly normal except for mild ptosis and hyperemic conjunctiva on the left side. Ophthalmic examination revealed normal intraocular pressure and normal eyeground. There were no specific findings in other physical examinations. Orbital CT revealed left orbital myositis involving superior, inferior, medial, and lateral rectus muscles compared with the right orbit (Fig. 1A). We treated him with intra-venous mannitol, $60 \mathrm{mg} /$ day per-oral prednisolone (nisolone $^{\circledR}$, Kukje, Seongnam, Korea), levofloxin eye-drops (Cravit $^{\circledR}$, Santen, Shiga, Japan), dorzolamide HCl $2 \%$ with timolol 0.5\% (Cosopt ${ }^{\circledR}$, MSD, Seoul, Korea), and latamoprost (Xalost $^{\circledR}$, Taejoon, Seoul, Korea).

After 3-days, he developed vesicular skin rashes at the distribution of the ophthalmic branch of the left trigeminal nerve and along the nasal ridge (Hutchinson's sign) (Fig. 1C). He complained of severe pain in his left forehead and nose. The pattern of the pain included lancinating, shooting, and itching sensations. The pain measured by the visual analogue scale (VAS) was approximately 90/100 mm. Laboratory data, including routine biochemical and hematological examinations, were normal except for glucose ( $423 \mathrm{mg} / \mathrm{dl}$ ) and hemoglobin Alc (10.6\%). The erythrocyte sedimentation rate (ESR) was $30 \mathrm{~mm} / \mathrm{hr}$ and C-reactive protein (CRP) was $0.74 \mathrm{mg} /$ dl. Immunologic studies, including IgA, IgM, C3, C4, antimitochondrial antibody (AMIA), anti-nuclear antibody (ANA), and anti-double stranded DNA, were all within normal limits. Thyroid function test was normal and the VDRL/TPHA test was negative.

Immediately, we treated him with 7-day intra-venous (IV) acyclovir, IV dexamethasone, and per-oral (PO) gabapentin. At the same time, we performed supraorbital and supratrochlear nerve blocks. After the nerve block, the headache and allodynia were improved. The eyelid swelling and orbital pain gradually lessened by the time he was discharged.

The patient returned to our clinic with persistent left orbital pain and paroxysmal and lancinating pain along the nose ridge and forehead. We performed supraorbital and supratrochlear nerve blocks then and again in two weeks, for a total of three times. The pain was reduced to VAS $10-20 / 100 \mathrm{~mm}$. A followup orbital CT revealed resolved myositis in the rectus muscles (Fig. 1B). The patient was undergoing oral administration of gabapentin ( $600 \mathrm{mg} /$ day). He revisited the hospital 5 months later without particular exacerbation of the pain or any other inconvenience in his daily tasks.

Ophthalmic complications following HZO result directly from inflammatory changes or nerve damage, or indirectly from tissue scarring. These complications vary from mild, which may pass unnoticed, to severe, which may threaten life or sight. Except for diplopia, our patient developed characteristic symptoms of orbital myositis, such as orbital pain worsening

Corresponding author: Hyung Tae Kim, M.D., Department of Anesthesiology and Pain Medicine, Presbyterian Medical Center, P.O.Box 77, Jeonju 560-750, Korea. Tel: 82-63-230-1593, Fax: 82-63-230-8128, E-mail: ingwei2475@gmail.com

(c) This is an open-access article distributed under the terms of the Creative Commons Attribution Non-Commercial License (http:// creativecommons.org/licenses/by-nc/3.0/), which permits unrestricted non-commercial use, distribution, and reproduction in any medium, provided the original work is properly cited. 

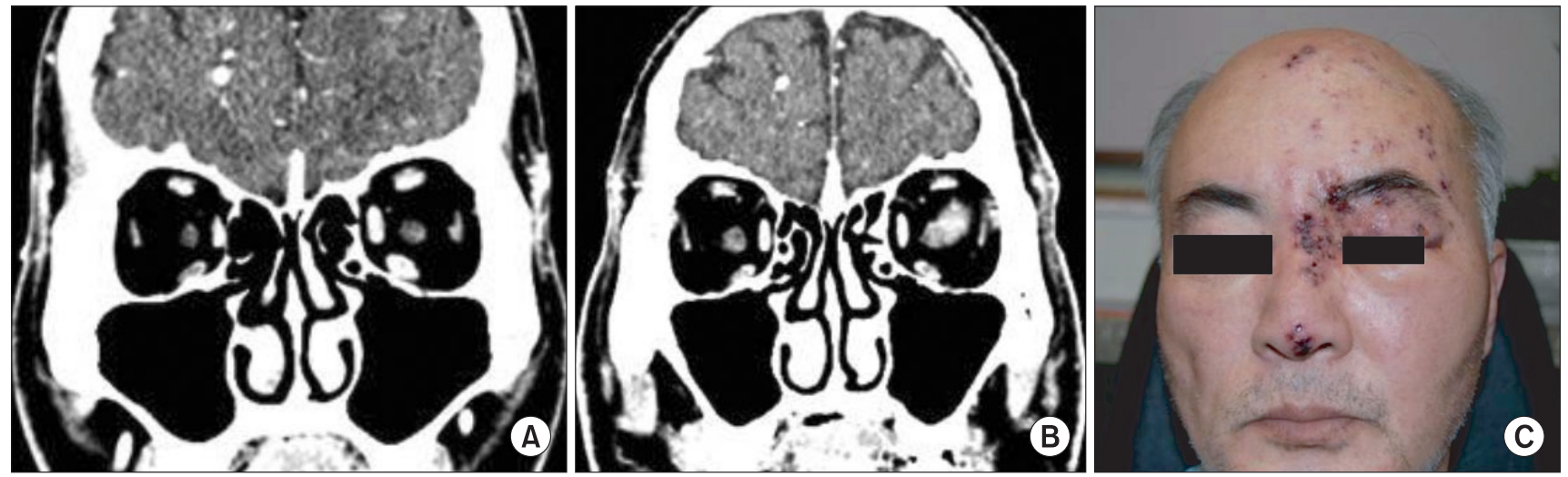

Fig. 1. (A) Orbital computed tomography (CT) image with contrast, coronal view. CT shows relatively moderately enhanced and enlarged left superior, inferior, medial, and lateral rectus muscles compared with right orbit. (B) 2 weeks later, a follow-up orbital CT image. CT shows symmetrical entire rectus muscles. (C) This patient developed vesicular skin eruptions at the ophthalmic branch of the left trigeminal nerve and along the nose ridge (Hutchinson's sign).

with eye movements, proptosis, swollen eyelid, and hyperemic conjunctiva. He had Hutchinson's sign, which is typical for HZO [1]. Normal immunologic and serologic surveys excluded other etiologies of orbital myositis, for example, thyroid disease, syphilis, and auto-immune diseases. His ocular symptoms improved after antiviral therapy and the follow-up orbital MRI four months later revealed total recovery of orbital myositis. Typically, ophthalmic complications of HZO occur between 5 days and 14 days following cutaneous lesions. Our case is unusual in that the orbital myositis preceded vesicular rashes. Two similar patients have been reported previously $[3,4]$. Both patients came to the hospital for retrobulbar pain with diplopia. Volpe et al. demonstrated orbital myositis on computed tomographic (CT) scans in their patient one day before the development of skin vesicles [3]. As reported by Kawasaki et al., MRI demonstrated orbital myositis three days prior to appearance of typical skin eruptions in their patient [4]. In these two patients and ours, their extraocular myositis had excellent recovery. However, our patient suffered from PHN, which did not occur in the previously reported patients. Although Marsh and Cooper had proposed extraocular myositis as a possible cause of ophthalmoplegia in HZO [2], it was not well documented until these case reports. To date, there is no histopathologic study of orbital myositis in HZO [4]. Orbital myositis preceding vesicular skin eruptions is a diagnostic challenge in HZO. Since zoster rashes may develop one week or more after dermatomal pain [1], serological and immunological tests may be helpful for early diagnosis in extraocular myositis preceding zoster rashes. In 1958, Lewis reported a syndrome of "ophthalmic zoster sine herpete" [5], in which orbital pain, extraocular palsy, and periorbital skin swelling occurred without skin rashes. "Ophthalmic zoster sine herpete" further confounds the diagnosis of HZO. We emphasize that, even without vesicular skin rashes, a diagnosis of extraocular myositis case as idiopathic should not be given before the availability of negative serological and immunological results for herpes zoster.

In conclusion, orbital myositis can be the presenting sign of HZO. In these patients, recovery of extraocular myositis is excellent and serological and immunological studies may be helpful for early diagnosis. HZO should be listed as a cause of acute orbital myositis even without skin eruptions. Therefore, early diagnosis of acute orbital myositis and anti-viral therapy will prevent PHN. And, if zoster skin rashes are found, we should control pain aggressively by nerve blocks to prevent the development to PHN.

\section{References}

1. McCrary ML, Severson J, Tyring SK. Varicella zoster virus. J Am Acad Dermatol 1999; 41: 1-14.

2. Marsh RJ, Cooper M. Ophthalmic herpes zoster. Eye (Lond) 1993; 7 : 350-70.

3. Volpe NJ, Shore JW. Orbital myositis associated with herpes zoster. Arch Ophthalmol 1991; 109: 471-2.

4. Kawasaki A, Borruat FX. An unusual presentation of herpes zoster ophthalmicus: orbital myositis preceding vesicular eruption. Am J Ophthalmol 2003; 136: 574-5.

5. Lewis GW. Zoster sine herpete. Br Med J 1958; 2: 418-21. 